This item was submitted to Loughborough's Research Repository by the author.

Items in Figshare are protected by copyright, with all rights reserved, unless otherwise indicated.

\title{
Electron-beam instability in left-handed media
}

PLEASE CITE THE PUBLISHED VERSION

http://dx.doi.org/10.1103/PhysRevLett.100.244803

PUBLISHER

(C) American Physical Society

VERSION

VoR (Version of Record)

LICENCE

CC BY-NC-ND 4.0

REPOSITORY RECORD

Bliokh, Yury P., Sergey Savel'ev, and Franco Nori. 2019. "Electron-beam Instability in Left-handed Media". figshare. https://hdl.handle.net/2134/12719. 
This item was submitted to Loughborough's Institutional Repository (https://dspace.lboro.ac.uk/) by the author and is made available under the following Creative Commons Licence conditions.

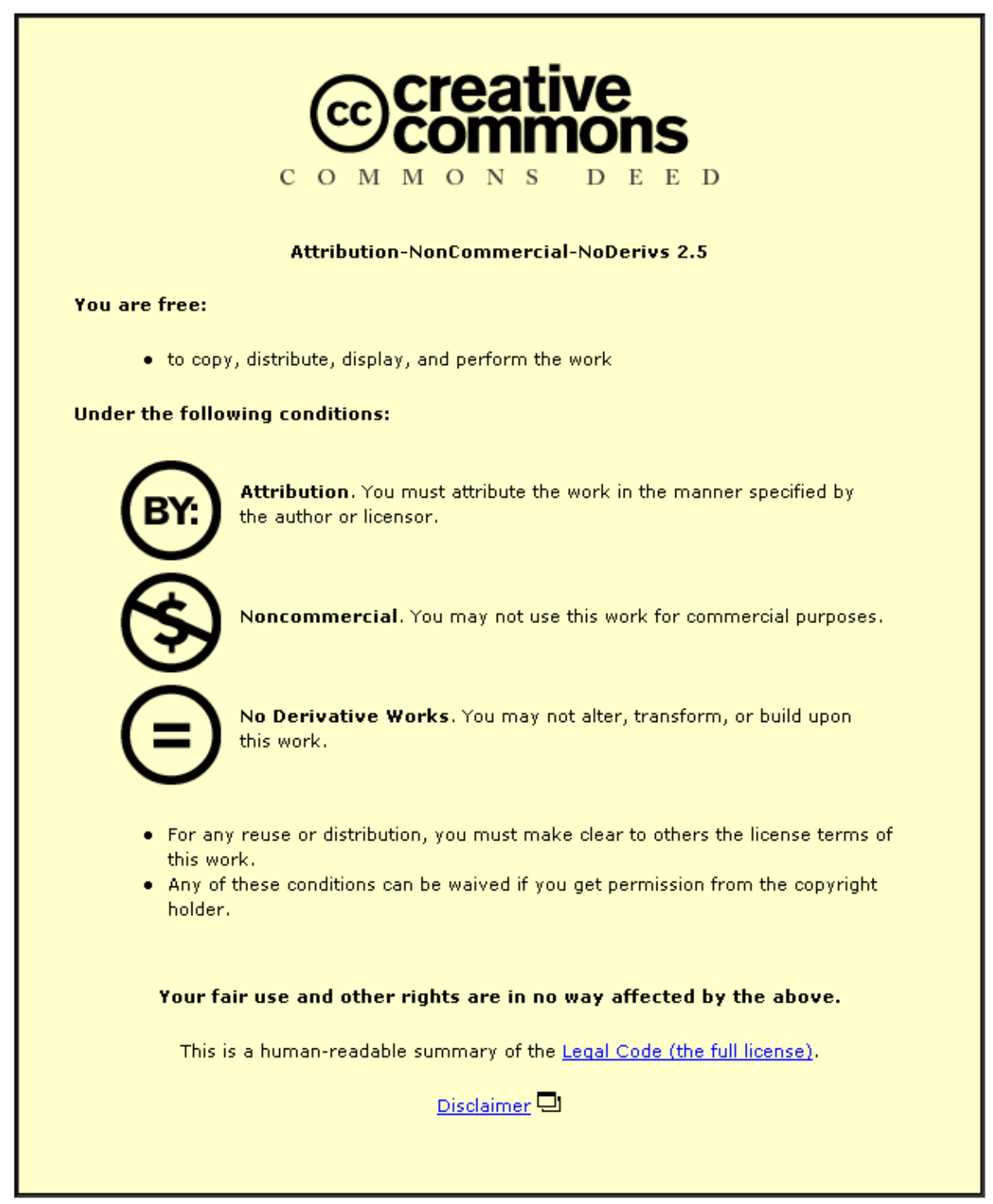

For the full text of this licence, please go to: http://creativecommons.org/licenses/by-nc-nd/2.5/ 


\title{
Electron-Beam Instability in Left-Handed Media
}

\author{
Yury P. Bliokh, ${ }^{1,2}$ Sergey Savel'ev, ${ }^{1,3}$ and Franco Nori ${ }^{1,4}$ \\ ${ }^{1}$ Advanced Science Institute, The Institute of Physical and Chemical Research (RIKEN), Wako-shi, Saitama 351-0198, Japan \\ ${ }^{2}$ Department of Physics, Technion-Israel Institute of Technology, Haifa 32000, Israel \\ ${ }^{3}$ Department of Physics, Loughborough University, Loughborough LE11 3TU, United Kingdom \\ ${ }^{4}$ Center for Theoretical Physics, Department of Physics, CSCS, University of Michigan, Ann Arbor, Michigan 48109-1040, USA
}

(Received 22 October 2007; published 19 June 2008)

\begin{abstract}
We predict that two electron beams can develop an instability when passing through a slab of lefthanded media (LHM). This instability, which is inherent only for LHM, originates from the backward Cherenkov radiation and results in a self-modulation of the beams and radiation of electromagnetic waves. These waves leave the sample via the rear surface of the slab (the beam injection plane) and form two shifted bright circles centered at the beams. A simulated spectrum of radiation has well-separated lines on top of a broad continuous spectrum, which indicates dynamical chaos in the system. The radiation intensity and its spectrum can be controlled either by the beams' current or by the distance between the two beams.
\end{abstract}

DOI: 10.1103/PhysRevLett.100.244803

PACS numbers: 41.75.- $\mathrm{i}, 41.60 . \mathrm{Bq}$

Metamaterials [also known as left-handed media (LHM) [1]], characterized by having negative both permittivity $\varepsilon$ and permeability $\mu$, show numerous remarkable counterintuitive features [2], including: negative refraction [1], inverted Doppler shift and reversed Cherenkov cone (backward Cherenkov radiation) [3], as well as surface waves propagating along the interface between right- and lefthanded media [4]. These properties (promising for applications in a wide frequency range for subwavelength imaging and lensing [5], subwavelength [6] and open [7] resonators [8], nonradiating configurations [9], etc.) have stimulated enormous scientific activity during the past decade. However, as far as we know, the collective interaction of charged particle beams with electromagnetic waves in LHM has not been studied. Nevertheless, one can expect a very nontrivial interaction among particles in the beams since the Cherenkov radiation emitted by a particle propagates backward $[3,10]$, producing strong positive distributed feedback for particles moving behind. Such a strong coupling can create an instability and chaotic (i.e., very irregular) motion in the beams and waves. In this article we predict the instability of electron beams in LHM associated with tunable self-sustained electromagnetic radiation.

In order to provide an intuitive picture of the effect discussed below, we compare information transport by electromagnetic waves (solid line) and particles (dashed line), shown in Fig. 1, for slabs of left-handed (a) and righthanded (b) media. Information about any perturbation created, for instance, at the rear (left) surface is transferred either along the wave characteristics (solid line) and/or along the particle trajectories (dashed line) deep towards the sample. For right-handed media (RHM), both information fluxes are directed forward and perturbations propagate within the shaded region between the characteristics [Fig. 1(b)]. Thus, any knowledge about the perturbation leaves the sample in the forward direction after a finite time. In contrast, for LHM, particles and waves transport information in opposite directions. Therefore, the information transported from the rear to the front surfaces by particles is returned back by the emitted waves [Fig. 1(a)]. Simultaneously, these "returning" waves perturb the particles entering the sample later on (in the rear surface), and the process of information transport will oscillate back and forth, and never stops. Similar processes occur in microwave devices as traveling wave tubes (TWT) (similar to a slab of RHM) and backward wave oscillators (BWO) (similar to a slab of LHM) [11].

It is well known [11] that positive feedback in BWO produces an electron-beam instability and self-excited microwave radiation. Thus, a similar instability can be expected in LHM. Consider, for example, a small perturbation (denoted here as "bunch") of the beam density with a longitudinal dimension smaller than the wavelength of the synchronous wave (i.e., a wave with phase velocity equal to the beam particles' velocity). Particles forming the bunch radiate coherently and the radiated electromagnetic field is not compensated (due to destructive interference) by other beam particles. This wave acts on electrons moving behind the bunch and periodically modulates their velocities. These velocity variations modulate the beam density resulting in secondary bunches in the beam. Because of the periodicity of the emitted waves, radiation produced by the bunches sum up coherently and the total field increases. A stronger field leads to a faster and deeper modulation of the beam density that, in turn, strengthens the field. As a result, the amplitude of the synchronous wave grows exponentially. This is the socalled beam instability, which has been discovered simultaneously in plasma physics $[12,13]$ and microwave electronics [14]. Thus, two collective effects (the coherent Cherenkov radiation of the particles forming the bunch 

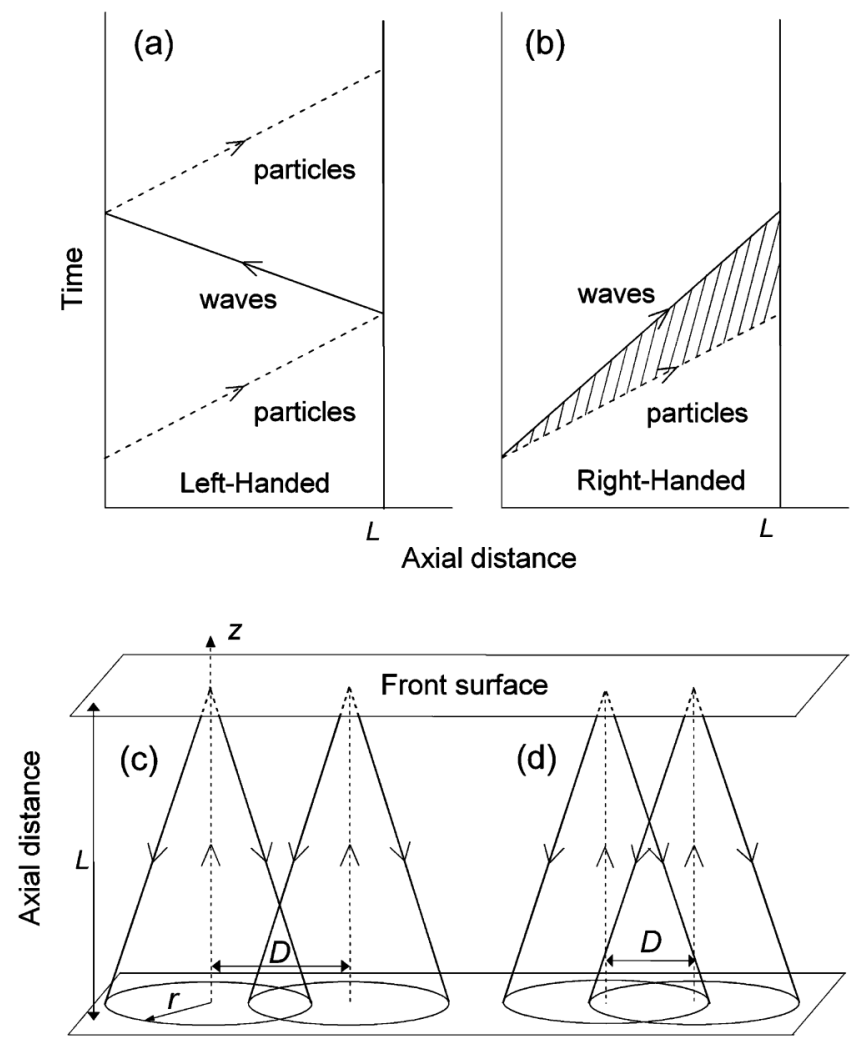

Rear surface

FIG. 1. Waves (solid line) and particles (dashed lines) characteristics in LHM (a) and RHM (b) slabs with thickness $L$. For LHM, particles and waves transport information in the opposite direction, while, for RHM, information can only be transmitted forward. (c),(d) Cherenkov field, emitted by particles (dashed lines), located within Cherenkov cones shown by solid lines. For case (c) the distance between the beams is too large, $D>D_{\max }$, and the radiation emitted by one beam cannot reach the other beam, so there is no interaction between beams. For case (d), $D<D_{\max }$, the radiation emitted by one beam reaches the tail of the other beam, creating a particle-density modulation producing a strong beam coupling.

and the coherence between the bunches) constitute the physical basis of the beam instability.

Here we consider two separated beams moving along a strong magnetic field and interacting via their Cherenkov radiations propagating backward in 3D LHM and predict a beam instability. This instability develops when the distance between the beams is within a certain interval and when the beam currents exceed a certain threshold.

Model. - We describe LHM by a frequency-dependent permittivity and permeability (see, e.g., $[5,9,15,16])$ :

$$
\varepsilon(\omega)=\mu(\omega)=1-\frac{\omega_{p}^{2}}{\omega^{2}+i \omega \nu},
$$

where $\nu$ is the collision frequency and $\omega_{p}$ is the plasma frequency. The medium is "left-handed" when the wave frequency $\omega<\omega_{p}$.
We consider two parallel beams, separated by a distance $D$ propagating along the $z$ direction (the limit $D \rightarrow 0$, corresponding to one beam, is discussed below). Charged particles in these beams emit Cherenkov radiation with the $z$ component of the electric field described by the standard formula [17]

$$
\begin{aligned}
E_{z 0}= & \frac{e}{c^{2}} \int\left(\frac{1}{n^{2} \beta^{2}}-1\right) J_{0}\left(\frac{\omega}{v} \sqrt{n^{2} \beta^{2}-1} r\right) \\
& \times \cos \left[\frac{\omega}{v}(z-v t)\right] \omega d \omega,
\end{aligned}
$$

where $v$ is the particle velocity, $\beta \equiv v / c$, and $n=-\sqrt{\varepsilon \mu}$ is the refraction index. The integration domain in a LHM is restricted by the conditions $\omega \geq 0,|n| \beta>1, \varepsilon<0$ and $\mu<0$ [i.e., $0 \leq \omega \leq \omega_{p} \beta /(1-\beta)$ ]. The other components of the radiated fields will not be needed because only the $E_{z}$-component governs the particle motion along the $z$ axis in a strong guiding magnetic field. Below we consider the nonrelativistic limit, $\beta \ll 1$, and small dissipation, $\nu \ll \beta^{1 / 2} \omega_{p}$. The motion of particles interacting through their emitted waves can be described by

$$
\frac{d^{2} z_{i}^{(1,2)}}{d t^{2}}=\frac{e}{m} \sum_{j}\left[E_{i j}^{(1,2)}+E_{i j}^{(2,1)}\right]
$$

where $z_{i}^{(k)}$ is the coordinate of the $i$ th particle in the $k$ th beam, $k=1,2$, and $E_{i j}^{(k)}=E_{z 0}\left[\left(z_{i}^{(\ell)}-z_{j r}^{(k)}\right)-\beta c(t-\right.$ $\left.\left.t_{j r}^{(k)}\right), r_{i j}\right]$ is the radiated field of the $j$ th particle from the $k$ th beam acting on the $i$ th particle from the $\ell$ th beam, $z_{j r}^{(k)}$ and $t_{j r}^{(k)}$ are the position and the time when the $j$ th particle radiated the wave that reaches the $i$ th particle at position $z_{i}$ at time $t, r_{i j}=0$ when $k=\ell$ and $r_{i j}=D$ otherwise. $E_{z 0}(z-\beta c t, r)$ is the "elementary" field with space-time structure defined by Eq. (2) and depicted in Fig. 2(a).

The essential property of the Cherenkov radiation emitted by a single particle is that the field is mostly concentrated along the line $\rho=\xi \tan \alpha$ and the angle $\alpha=$ $\arcsin (1 / 3)$ is independent of the particle velocity; in full analogy with the wake that a moving ship produces on the surface of the sea [18]. This means that we can associate a unique group velocity $v_{g}$ to the largest fraction of the radiation. Thus, we can approximate the position $z_{j r}$ and the time $t_{j r}$ as

$t_{j r}=t_{j 0}+\int_{0}^{z_{j r}} \frac{d z^{\prime}}{v_{j}\left(z^{\prime}\right)}, \quad z_{j r}=z+\left|v_{g z}\right|\left(t-t_{j r}\right) \cos \alpha$,

where $t_{j 0}$ is the time when the particle crosses the rear surface $z=0$ of the LHM slab.

Hereafter the following dimensionless variables are used: $\xi=k_{0 z} z, \eta=k_{0 z} x, \zeta=k_{0 z} y$, and $\tau=k_{0 z} v_{0} t\left(v_{0}\right.$ is the unperturbed beam velocity). 


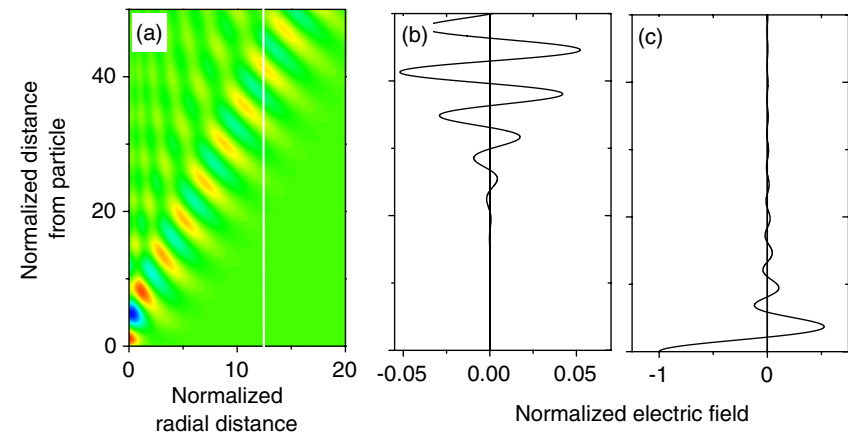

FIG. 2 (color online). Spatial distribution of the Cherenkov radiation emitted by a single particle. (a) The field distribution $E_{z}$ in the $\left(\rho, \xi^{\prime}\right)$-plane, where $\rho=k_{0 z} r$ and $\xi^{\prime}=k_{0 z}(z-v t)$ are the normalized radial and axial distances from the particle; $k_{0 z}$ is the axial component of the wave vector $\vec{k}_{0}$ corresponding to the maximum of the spectrum of radiation. The electric field amplitude is depicted with the factor $\sqrt{\rho}$, which compensates the field decay $E \sim \rho^{-1 / 2}$. (b), (c): axial distribution of the field $E_{z}$ for $\rho=12.5$ [white line in Fig. 2(a)] and $\rho=0$ accordingly. Note that $E_{z}(\xi)$ for $\rho=12.5$ has the form of a wave packet with a distinguishable periodicity (b), whereas $E_{z}(\xi)$ for $\rho=0$ is fastdecaying and nonperiodic (c).

Instability of two beams. - We simulated the motion of electrons in two thin beams, separated by a distance $D$ in the transverse direction, using Eqs. (3) and (4); each simulation takes time $\tau_{s} \gg \mathcal{L}$, where $\mathcal{L}=k_{0 z} L$ is the dimensionless thickness of the slab. The simulations showed that, at certain conditions, a beam instability develops: any small perturbation develops into a strong modulation of the beam density. Inside the slab, the beam modulation increases strongly away from the rear surface and exhibits a maximum approaching the front surface [Fig. 3(a)]. The beam density modulation is the source of radiation which is transported along the reverse Cherenkov cone [see Fig. 1(c), 1(d), and 2(a)] by the backward waves to the rear surface. The total field $E_{z}$ is calculated as a sum of the elementary fields radiated by all the particles inside the slab. The total radiation intensity, $I_{\text {tot }}=$ $\int d \eta d \zeta I(\eta, \zeta, \xi=0)=\tau_{s}^{-1} \int d \eta d \zeta d \tau E_{z}^{2}(\xi=0, \eta, \zeta, \tau)$ is not zero when the beam separation $\Delta=k_{0 z} D$ is within a certain interval $\Delta \in\left(\Delta_{\min }, \Delta_{\max }\right)$ [Fig. 3(b)]. The window $\left(\Delta_{\min }, \Delta_{\max }\right)$ of this instability weakly depends on the beam current (for simplicity, we consider two beams with the same current $J_{b}$ ). The spatial distribution of the radiation intensity $I(\eta, \zeta)$ at the rear surface forms two overlapping or intersecting rings, as shown in Fig. 3(c). These bright rings could be seen by an observer located outside the slab.

The physical origin of the suppression of the instability for any large separation distance $\Delta>\Delta_{\max }$ is rather obvious: indeed, in this case the neighboring beams are located outside their corresponding Cherenkov cones and cannot interact via their emitted waves [Fig. 1(c)]. At small distances $\Delta<\Delta_{\min }$, the instability is suppressed due to three different reasons. First, the electromagnetic field
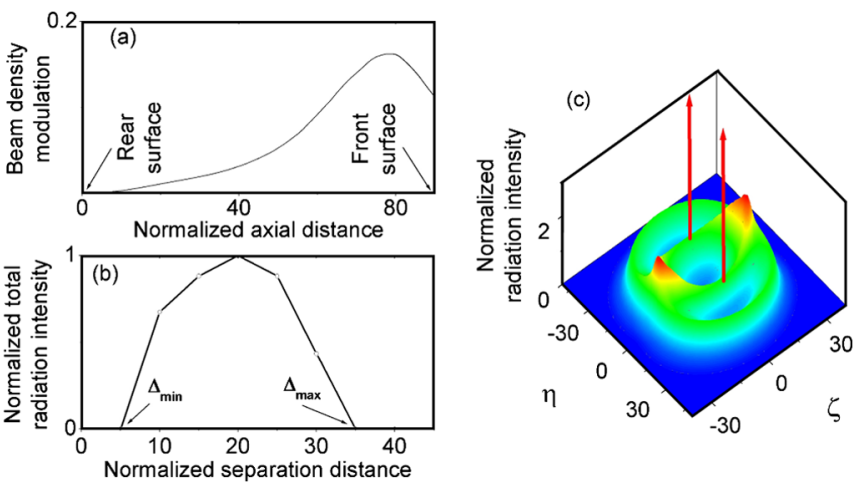

FIG. 3 (color online). (a) Amplitude of the beam density modulation (due to bunching of particles) along the beam, divided by the average density. The maximum of the density modulation occurs near the front surface; thus, beams radiate with higher intensity near this surface. (b) Total radiation intensity $I_{\text {tot }}$ versus the separation distance $\Delta$ between two beams. This intensity is nonzero for $\Delta_{\min }<\Delta<\Delta_{\max }$. (c) Spatial distribution of the radiation intensity $I(\eta, \zeta)$ at the rear surface of the LHM slab. This distribution has the form of two shifted circles centered at the beams.

emitted by a particle decreases fast [see Fig. 2(c)] along the beam. Second, the spatial period of the decaying wave varies behind the particle, which destroys the spatial periodicity of the beam modulation and, as a result, suppresses the coherence of the radiation emitted by this modulation. These two reasons are common for the destruction of the beam instability in both 3D LHM and BWO and explain why there is no instability in the one-beam system. The other, third reason, is unique for 3D LHM: when the distance between the beams decreases, the region of intersection of one beam with the Cherenkov cone formed by the second beam is shifted toward the front surface of the slab. The beam modulation is amplified along the distance between this intersection and the front surface of the slab. This distance is shortened when the beam separation decreases and becomes insufficiently long for an instability to develop. Particles in the two different beams interact effectively and the radiation intensity reaches its maximum when the intersection between the beam and the Cherenkov cone occurs near the rear surface [Fig. 1(d)]. This is in agreement with our simulations [Fig. 3(a)]. Therefore the distance between the beams can effectively control the intensity of the radiation.

Properties of radiation. - Let us consider the radiation for the optimal separation between the beams. An analogy with BWO [11] points out that we can expect three different radiation regimes: (i) no radiation for either a weak beam current or small slab thickness, (ii) monochromatic radiation above a certain current or thickness thresholds, and (iii) periodic or chaotic self-modulation at larger currents or thicknesses. Indeed, we found no instability at either small current or thin samples. Above a threshold we always observed a chaotic self-modulation. An ex- 

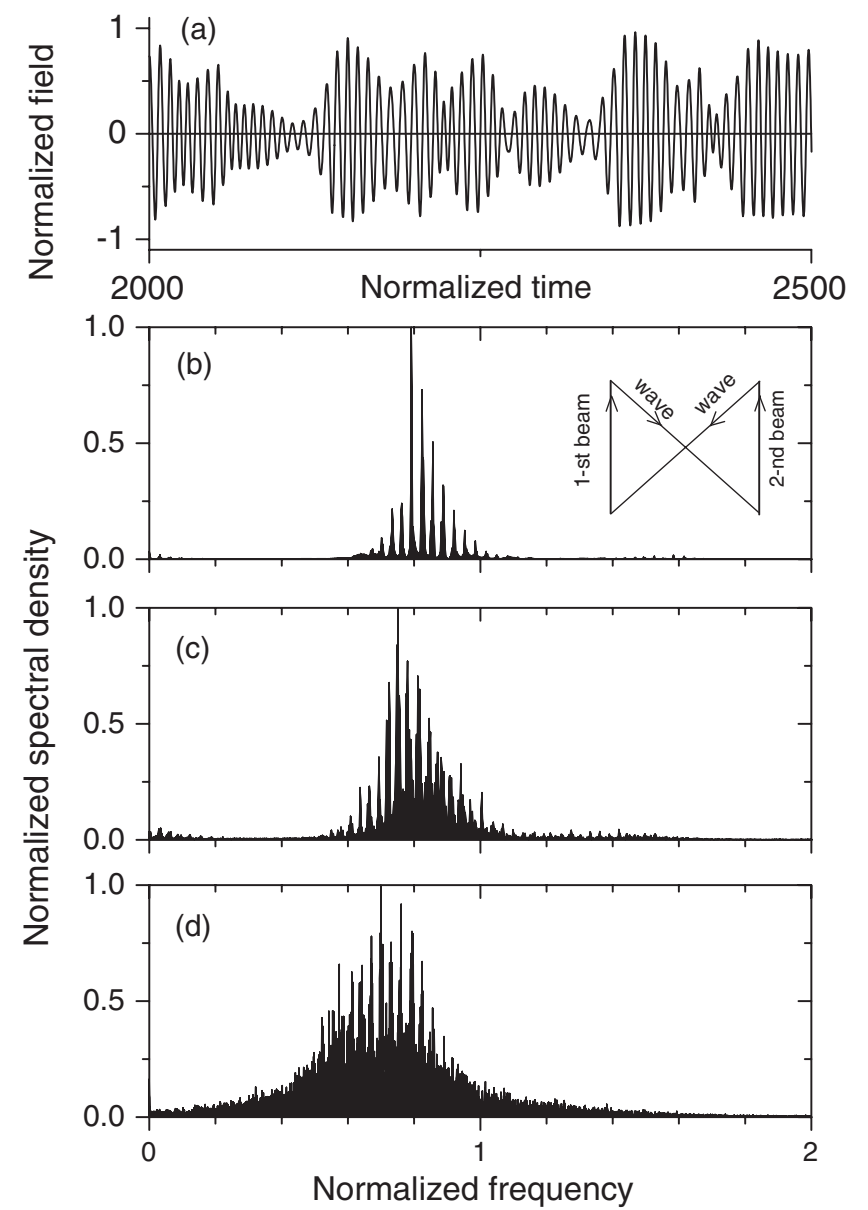

FIG. 4. (a) Time dependence of the electric field $E(\tau)$ of the emitted wave at the rear surface of the LHM slab. The irregular modulation of the wave amplitude indicates chaotic dynamics of the beams. (b) -(d) Radiation spectra of a two-beam system when the beam current grows. Spectra show well-separated narrow lines on top of a continuous background. The current increases by a factor 1.5 from (b) to (d).

ample of the time-dependence of the electric field of the emitted wave at the rear surface is presented in Fig. 4(a). This dependence clearly shows the irregular modulation of the wave amplitude. The spectra of the wave is shown in Fig. 4(b)-4(d), for increasing beam current. All of these three presented spectra have a similar structure: narrow spectral lines whose overlap forms a continuous spectrum. With increasing current, the lines become broader and the strength of the continuous spectrum grows, which is usual for microwave devices with a so-called "frequency" scenario of transition to chaos [19]. The frequency gap between the spectral lines is determined by the time needed for a signal to close the feedback loop [signal is transported first by one beam, then the signal is carried back by the electromagnetic wave from the beam head to the tail of the second beam, then forward along the second beam, and afterward by the wave from the head of the second beam to the initial point; see inset in Fig. 4(b)]. Therefore, the distance between the spectral lines can be tuned by the distance between the beams or can be designed by a proper choice of the slab thickness.

Conclusions. - We predict a beam instability in 3D lefthanded media. This instability produces strong radiation which can be tuned either by the beam current or by geometrical parameters. The spectrum of the radiation has well-separated lines on top of a broad background. Since now LHM can be fabricated [20] in the optical frequency range, the predicted effect can be used to generate and amplify optical irregular signals. Further extension of the analogy between traditional microwave devices and left-handed media will allow new insights for new potential applications of LHM.

We acknowledge partial support from the NSA, LPS, ARO, NSF Grant No. EIA-0130383, JSPS-RFBR No. 0602-91200, MEXT Grant-in-Aid No. 18740224, the EPSRC via No. EP/D072581/1 and No. EP/F005482/1, the AQDJJ ESF network-program, and the JSPS CTC Program.

[1] V. G. Veselago, Sov. Phys. Usp. 10, 509 (1968).

[2] D. R. Smith et al., Science 305, 788 (2004); D. R. Smith, Science 308, 502 (2005); V. G. Veselago and E. E. Narimanov, Nat. Mater. 5, 759 (2006); V. M. Shalaev, Nat. Photon. 1, 41 (2007).

[3] V.E. Pafomov, Sov. Phys. JETP 9, 1321 (1959).

[4] R. Ruppin, J. Phys. Condens. Matter 13, 1811 (2001); A. V. Kats et al., Phys. Rev. Lett. 98, 073901 (2007).

[5] J. B. Pendry, Phys. Rev. Lett. 85, 3966 (2000).

[6] N. Engheta, IEEE Antennas Wireless Propagat. Lett. 1, 10 (2002).

[7] M. Notomi, Phys. Rev. B 62, 10696 (2000).

[8] K. Yu. Bliokh et al., arXiv:0708.2653 [Rev. Mod. Phys. (to be published)].

[9] A. D. Boardman and K. Marinov, J. Opt. Soc. Am. B 23, 543 (2006).

[10] J. Lu et al., Opt. Express 11, 723 (2003); C. Luo et al., Science 299, 368 (2003); M. Cheng, Opt. Express 15, 9793 (2007).

[11] A. S. Gilmour, Microwave Tubes (Artech House, Dedham, MA, 1986).

[12] A. I. Akhieser and Ya. B. Fainberg, Dokl. Akad. Nauk SSSR 119, 555 (1949).

[13] D. Bohm and E. P. Gross, Phys. Rev. 75, 1851 (1949).

[14] J. R. Pierce, J. Appl. Phys. 20, 1060 (1949).

[15] R. W. Ziolkovski and E. Heyman, Phys. Rev. E 64, 056625 (2001).

[16] S. A. Ranmakrishna, Rep. Prog. Phys. 68, 449 (2005).

[17] J. V. Jelly, Cerenkov Radiation and its Applications (Pergamon, London, 1958).

[18] M. J. Lighthill, Waves in Fluids (Cambridge University Press, Cambridge, England, 1978).

[19] Yu. P. Bliokh et al., Appl. Nonlinear Dynamics 1, 34 (1993); Yu. P. Bliokh et al., Chaos Solitons Fractals 7, 273 (1996).

[20] A. N. Grigorenko et al., Nature (London) 438, 335 (2005). 